\title{
CANTOR SINGULAR CONTINUOUS SPECTRUM FOR OPERATORS ALONG INTERVAL EXCHANGE TRANSFORMATIONS
}

\author{
M. COBO, C. GUTIERREZ, AND C. R. DE OLIVEIRA \\ (Communicated by Joseph A. Ball)
}

\begin{abstract}
It is shown that Schrödinger operators, with potentials along the shift embedding of Lebesgue almost every interval exchange transformations, have Cantor spectrum of measure zero and pure singular continuous for Lebesgue almost all points of the interval.
\end{abstract}

\section{INTRODUCTION AND MAIN RESULT}

In [7] the spectrum $\sigma\left(H_{\omega}\right)$ of the discrete Schrödinger operators

$$
\begin{aligned}
& H_{\omega}: l^{2}(\mathbb{Z}) \rightarrow l^{2}(\mathbb{Z}), \\
& \left(H_{\omega} \psi\right)_{j}:=\psi_{j+1}+\psi_{j-1}+\omega_{j} \psi_{j},
\end{aligned}
$$

with $\omega=\left(\omega_{j}\right)_{j \in \mathbb{Z}}$ a sequence of real numbers (the so-called potential) modulated along the shift embedding of interval exchange transformations (iets) [10, 11], was investigated. There the authors proved the presence of the pure singular continuous spectrum for $H_{\omega}$, for the shift associated with a dense set of iets, where the potential $\omega$ corresponds to the itinerary of the orbit of a.e. $x$ in the interval (from here on, a.e. with no specification means almost everywhere with respect to Lebesgue measure).

In this work we go a step further by showing that the above mentioned results of [7] hold for Lebesgue almost every iets. The proof of absence of eigenvalues involves a rather different argument, mainly the Rauzy induction map. As a byproduct of our a.e. results, which are summarized in Theorem 1 below, there is a set of interesting results in the literature ready to be applied, which leads to Cantor spectrum of zero Lebesgue measure; see Corollary 1.

Interval exchanges. Let us recall some notation and a description of the iets necessary to state and prove our results.

Given a semi-open interval $[a, b)$ and a vector $\lambda=\left(\lambda_{1}, \lambda_{2}, \ldots, \lambda_{n}\right)$ in $\mathbb{R}_{+}^{n}$ (i.e., all entries are positive) such that $\lambda_{1}+\lambda_{2}+\cdots+\lambda_{n}=b-a$, we consider the partition of $[a, b)$ in the $n$ consecutive semi-open intervals

$$
I_{1}:=\left[a, a_{1}\right), I_{2}:=\left[a_{1}, a_{2}\right), \ldots, I_{n}:=\left[a_{n-1}, b\right)
$$

Received by the editors June 12, 2006 and, in revised form, October 23, 2006.

2000 Mathematics Subject Classification. Primary 47B36, 47B37, 37B05, 37B10.

Key words and phrases. Schrödinger operator, interval exchange transformation, singular continuous spectrum, Cantor spectrum. 
whose lengths are respectively $\lambda_{1}, \lambda_{2}, \ldots, \lambda_{n}$. Let $\pi$ be a permutation of the symbols $\{1,2, \ldots, n\}$. An iet $E:[a, b) \rightarrow[a, b)$ is associated to the pair $(\pi, \lambda)$ by exchanging the positions of the intervals $I_{i}$ according to the permutation $\pi$, in such a way that the interval in the $i^{t h}$ position, $I_{i}$, is translated to the $\pi(i)^{t h}$ position (from left to right). In this way the transformation obtained is of the form

$$
E(x)=x+d_{i}, \quad x \in I_{i}, \quad i=1,2, \ldots, n,
$$

for some displacements $d_{1}, \ldots, d_{n}$.

In this work we consider mostly iets of the form $E:[0, b) \rightarrow[0, b), b>0$. An iet $E$ is normalized if it is defined in the interval $[0,1)$. The set of all normalized iets is parameterized in the following way. Let $\Delta^{n-1}$ denote the standard simplex of $\mathbb{R}^{n}$,

$$
\Delta^{n-1}:=\left\{\left(\lambda_{1}, \lambda_{2}, \ldots, \lambda_{n}\right) \in \mathbb{R}_{+}^{n}: \lambda_{1}+\lambda_{2}+\cdots+\lambda_{n}=1\right\} .
$$

If $P_{n}$ is the set of all permutations of the symbols $\{1,2, \ldots, n\}$, then it is usual to identify the product $P_{n} \times \Delta^{n-1}$ with the set of all normalized interval exchanges of $n$ intervals. Let us denote by $G_{n}$ the set of irreducible permutations in $P_{n}$, i.e., those permutations $\pi$ for which $\pi(\{1,2, \ldots, k\}) \neq\{1,2, \ldots, k\}$ unless $k=n$. For a fixed permutation $\pi \in G_{n}$ we denote by $E(\pi)$ the set of all normalized iets with permutation $\pi$. We identify the metric spaces $\Delta^{n-1}$ and $E(\pi)$ by the homeomorphism $\Delta^{n-1} \ni \lambda \mapsto E_{\lambda}:=(\pi, \lambda)$.

Let $\Sigma_{n}:=\{1,2, \ldots, n\}^{\mathbb{Z}}$. Associated to the orbit of $x$ by $E_{\lambda}$ there is the so-called itinerary of $x, \omega_{\lambda}(x)$, which is an element of $\Sigma_{n}$ and which is given by the natural encoding of the $E_{\lambda}$-orbit of $x$ by assigning to each entry of this orbit the suffix $i$ of the interval $I_{i}$ which contains it. Set

$$
\Omega_{\lambda}=\text { closure }\left\{\omega_{\lambda}([0,1))\right\}
$$

in $\Sigma_{n}$, so that $\Omega_{\lambda}$ with the left shift dynamics is a subshift over the alphabet $\{1,2, \ldots, n\}$.

In the next theorem we will consider the $(n-1)$-dimensional Lebesgue measure in $\Delta^{n-1} \cong E(\pi), \pi \in G_{n}$. We also recall that a nonempty set in a metric space is a Cantor set if it is closed with empty interior and no isolated points.

Let us fix an irreducible permutation $\pi \in G_{n}$ and an arbitrary injective map $V$ : $\{1,2, \ldots, n\} \rightarrow \mathbb{R}$. For any $\omega \in \Sigma_{n}$ consider the potential $V(\omega):=\left(V\left(\omega_{j}\right)\right)_{j \in \mathbb{Z}}$ and the operator $H_{V(\omega)}$ as in (1).

Theorem 1. There is a subset $\mathcal{F} \subset E(\pi)$ of full Lebesgue measure such that, for each $E_{\lambda} \in \mathcal{F}$, the spectrum of the corresponding Schrödinger operator (1) with potential $V\left(\omega_{\lambda}(x)\right)$ is a Cantor set of zero (Lebesgue) measure and pure singular continuous for a.e. $x \in[0,1)$.

We recall that for $n=2$ and $\pi(1,2)=(2,1)$ there is only one discontinuity point $a_{1} \in[0,1)$ and the system is reduced to rotations of the circle by the angle (1$\left.a_{1}\right)$. In this case the potentials $\Omega_{E}$ are the Sturmian sequences $[1,5]$, which include the well-known Fibonacci substitution sequence [14, 17]. Hence, the potentials generated by iets are natural generalizations of Sturmian potentials, one of the standard models of one-dimensional quasicrystals. We refer the reader to [7] for additional comments and to [6], as well as references therein, for related examples of Cantor zero measure spectrum.

We close this section with an open question we have found interesting. Although almost all iets are minimal and uniquely ergodic $[13,18]$, there are cases of minimal 
iets with more than one ergodic component ( $n / 2$ is an upper bound for the number of ergodic probability measures $[11,13])$, so it is natural to ask if the characterization of the spectrum in Theorem 1 holds in such cases.

\section{Some RELATED RESUlts}

The proof of Theorem 1 will be reduced to the proof that, given an irreducible permutation $\pi \in G_{n}$, for almost every iet $E_{\lambda} \in E(\pi)$, the corresponding Schrödinger operators $H_{V\left(\omega_{\lambda}(x)\right)}$ have no eigenvalues for Lebesgue almost every $x \in[0,1)$. In this section we clarify this statement and the next sections are devoted to the related proof of the absence of eigenvalues.

Consider a finite alphabet $\mathcal{A}$ and let $W$ be the set of finite words in this alphabet. If $B \in W$ we denote by $|B|$ its length. Consider a subshift $\Omega$ over $\mathcal{A}$ (the dynamics is always given by the left shift) and, for a given word $B \in W$, let us denote by

$$
V_{B}=V_{B}(\Omega)=\{\omega \in \Omega: \omega(1) \cdots \omega(|B|)=B\},
$$

the cylinders. For each invariant probability measure $\mu$ on $\Omega$, set

$$
\eta_{\mu}(n)=\min \left\{\mu\left(V_{B}\right): B \in W,|B|=n\right\} .
$$

In $[2,3]$ Boshernitzan introduced the following condition (which was later called condition (B) in $[6]$ ): the subshift $\Omega$ satisfies condition (B) if there exists an ergodic probability measure $\mu$ on $\Omega$ with

$$
\limsup _{n \rightarrow \infty} n \eta_{\mu}(n)>0 .
$$

This condition was shown by Boshernitzan [4] to imply unique ergodicity for minimal subshifts and, in the particular case of iets, was previously done by Veech [19]. Boshernitzan [4] proved

Theorem 2. Let $\pi \in G_{n}$. Then for Lebesgue almost every $\lambda \in \Delta^{n-1}$ the subshift $\Omega_{\lambda}$ satisfies condition (B).

Then, Veech [18] and Masur [13] obtained the result that $E_{\lambda}$ is uniquely ergodic (and, in particular, minimal) for a.e. $\lambda \in \Delta^{n-1}$.

Recall also the following basic result:

Lemma 1 ([10]). If $E_{\lambda}$ is minimal, then $\Omega_{\lambda}$ is a minimal subshift, i.e., every $\omega \in \Omega_{\lambda}$ has dense orbit in $\Omega_{\lambda}$.

With respect to the spectrum of discrete Schrödinger operators, the following important result was proved in [6]:

Theorem 3. Let $\Omega$ be a minimal subshift which satisfies condition (B). If $\Omega$ is aperiodic, then there exists a Cantor set $\Sigma \subset \mathbb{R}$ of Lebesgue measure zero so that the spectrum $\sigma\left(H_{\omega}\right)=\Sigma$ for every $\omega \in \Omega$.

It is well known that minimality implies that the spectrum (as a set) is the same for all elements in the hull $\Omega$; by combining this with Theorem 3 and the constancy of the absolutely continuous spectrum [12], for a fixed injective map $V:\{1,2, \ldots, n\} \rightarrow \mathbb{R}$ we summarize some important known results in

Corollary 1. Let $\pi \in G_{n}$. There is a subset $\mathcal{L} \subset E(\pi)$ of full Lebesgue measure so that: 
(i) for each $E_{\lambda} \in \mathcal{L}$ the spectrum of $H_{V(\omega)}$ in (1) is the same for all $\omega \in \Omega_{\lambda}$, and it is a Cantor set of zero Lebesgue measure;

(ii) for each $E_{\lambda} \in \mathcal{L}$ the corresponding Schrödinger operators (1) with potentials $V\left(\omega_{\lambda}(x)\right)$ have no absolutely continuous spectrum for all $x \in[0,1)$.

Therefore in order to prove Theorem 1 it is enough to show that, given $\pi \in G_{n}$, there is a set $\mathcal{P} \subset E(\pi)$ of full Lebesgue measure so that for each $E_{\lambda} \in \mathcal{P}$ the corresponding Schrödinger operators $H_{V\left(\omega_{\lambda}(x)\right)}$ have no eigenvalues for Lebesgue almost every $x \in[0,1)$; thus only a singular continuous spectrum remains. Then the set $\mathcal{F}$ in Theorem 1 can be defined by the intersection of this set $\mathcal{P}$ with $\mathcal{L}$.

An important tool to exclude eigenvalues for a given operator $H_{\omega}, \omega \in \Sigma_{n}$, is the Delyon-Petritis [8] version of an argument of Gordon [9], by means of suitable local word repetitions.

Theorem 4 ([8]). If for given $\omega \in \Sigma_{n}$ there exists a sequence $k_{i} \rightarrow \infty$ such that

$$
\omega_{j-k_{i}}=\omega_{j}=\omega_{j+k_{i}}
$$

for all $1 \leq j \leq k_{i}$, then the Schrödinger operator $H_{\omega}$ in (1) has no eigenvalues.

Given an irreducible permutation $\pi$, the idea is to show that, for almost all $\lambda \in$ $\Delta^{n-1}$, Theorem 4 applies to $H_{\omega}, \omega=\omega_{\lambda}(x)$, with $x$ in a set of total Lebesgue measure over $[0,1)$. In other words, we have to prove that for almost all $x \in[0,1)$ there is a sequence of natural numbers $r_{k} \rightarrow \infty$ such that the itinerary of $x$ associated to $\left[-r_{k}, 2 r_{k}\right]$ (i.e., the itinerary of the finite orbit $\left.E_{\lambda}^{-r_{k}}(x), E_{\lambda}^{-r_{k}+1}(x), \ldots, E_{\lambda}^{2 r_{k}}(x)\right)$ is of the form

$$
\overbrace{\omega_{0} \omega_{1} \ldots \omega_{r_{k}}} \overbrace{\omega_{0} \omega_{1} \ldots \omega_{r_{k}}} \overbrace{\omega_{0} \omega_{1} \ldots \omega_{r_{k}}}, \quad \omega_{i} \in\{1,2, \ldots, n\} .
$$

To prove this statement (which is Proposition 1 of Section 4) we will use the wellknown Rauzy Renormalization Operator in the space of interval exchange transformations. In the next section we will introduce the appropriate definitions and necessary results.

\section{RAUZY'S RENORMALIZATION}

Let $E:[a, b) \rightarrow[a, b)$ be a minimal iet such that $E=(\lambda, \pi)$ for some $\pi \in G_{n}$ and $\lambda \in \mathbb{R}_{+}^{n}$. Let $J:=[c, d)$ be a proper subinterval of $[a, b)$. Let us denote by $E_{J}$ the Poincaré's first return map of $E$ to the interval $J$, that is, for $x \in J, E_{J}(x)$ is given by the first point in the positive orbit of $x$ (by $E$ ) that steps into the interval $J$. By the minimality of $E$, the map $E_{J}$ is again an iet of $p$ intervals with $p \geq n$. We will be interested only in the case $p=n$; in this way $E_{J}$ will be associated to a pair $\left(\lambda^{\prime}, \pi^{\prime}\right)$ with $\lambda^{\prime} \in \mathbb{R}_{+}^{n}$ and $\pi^{\prime} \in G_{n}$. The iet $E_{J}$ will be called the induced map of $E$ onto the interval $J$. Let $I_{1}, I_{2}, \ldots, I_{n}$ be the intervals of continuity of $E_{J}$. For each $1 \leq k \leq n$ there exists an integer $r_{k}>0$ such that

$$
E\left(I_{k}\right), E^{2}\left(I_{k}\right), \ldots, E^{r_{k}}\left(I_{k}\right)
$$

are all intervals disjoint of $J$ whereas $E^{r_{k}+1}\left(I_{k}\right)$ is totally contained in $J$. By definition $E_{J}\left(I_{k}\right)=E^{r_{k}+1}\left(I_{k}\right)$. The number $r_{k}$ is called the return time of $I_{k}$ to $J$. We remark that the minimality of $E$ also implies that the interval $[a, b)$ is given by the union

$$
[a, b)=\bigcup_{k=1}^{n} \bigcup_{j=1}^{r_{k}} E^{j}\left(I_{k}\right)
$$


Rauzy's map. Take $(\pi, \lambda) \in G_{n} \times \Delta^{n-1}$ and let $E$ be the $(\pi, \lambda)$-interval exchange. Consider $\nu=\nu(\pi, \lambda)$ defined as the minimum between $\lambda_{n}$ and $\lambda_{\pi^{-1}(n)}$ provided that these numbers are different. If $E_{J}$ is the induced map of $E$ onto the interval $J:=[0,1-\nu), \nu>0$, it is proved in $[15,16]$ that $E_{J}$ is an iet of exactly $n$ intervals associated to a new irreducible permutation $\pi^{\prime} \in G_{n}$ and some vector in $\mathbb{R}_{+}^{n}$. Then, by normalizing $E_{J}$, we obtain a pair $\left(\pi^{\prime}, \lambda^{\prime}\right) \in G_{n} \times \Delta^{n-1}$. Rauzy's Renormalization map is the association $(\pi, \lambda) \stackrel{R}{\rightarrow}\left(\pi^{\prime}, \lambda^{\prime}\right)$. We remark that such association is not defined when $\lambda_{n}=\lambda_{\pi^{-1}(n)}$. As a consequence of this, when all iterates $R^{n}$ of the Rauzy map $R$ are defined in an iet $E_{\lambda}=(\pi, \lambda)$, then the orbits of any pair of discontinuity points of $E_{\lambda}$ are disjoint and so, by a Keane's result [10], $E_{\lambda}$ is minimal.

It is proved in [15] that $G_{n}$ is divided into several subsets called Rauzy Classes which are invariant by the process of induction just defined. Let us denote by $\mathcal{C}$ one of the Rauzy classes of $G_{n}$. Then if $\pi \in \mathcal{C}$ and $R(\pi, \lambda)=\left(\pi^{\prime}, \lambda^{\prime}\right)$, then $\pi^{\prime} \in \mathcal{C}$. Set

$$
\Delta_{\mathcal{C}}^{n-1}:=\left\{\left(\lambda_{1}, \lambda_{2}, \ldots, \lambda_{n}\right) \in \Delta^{n-1}: \lambda_{n} \neq \lambda_{\pi^{-1}(n)}, \pi \in \mathcal{C}\right\} .
$$

Then the transformation $R(\pi, \lambda)=\left(\pi^{\prime}, \lambda^{\prime}\right)$ is well defined in $\mathcal{C} \times \Delta_{\mathcal{C}}^{n-1}$. In $\mathcal{C} \times \Delta^{n-1}$ there is a natural measure $m$ which is the product of the counting measure in $\mathcal{C}$ and the $(n-1)$-dimensional Lebesgue measure in $\Delta^{n-1}$. As the set $\Delta_{\mathcal{C}}^{n-1}$ has total measure in $\Delta^{n-1}$ (regarding the $(n-1)$-dimensional Lebesgue measure), the map $R$ is defined $m$-almost everywhere in $\mathcal{C} \times \Delta^{n-1}$ and by abuse of language it is usually written

$$
R: \mathcal{C} \times \Delta^{n-1} \rightarrow \mathcal{C} \times \Delta^{n-1} .
$$

Rauzy's transformation plays a central role in the ergodic theory of iets due to the following result $[18,13]$.

Theorem 5. Rauzy's operator is ergodic for a measure which is absolutely continuous with respect to the probability measure $m$ defined above.

Another related result proved in $[18,13]$ is

Theorem 6. For a fixed permutation $\pi \in \mathcal{C}$ and for almost every vector $\lambda \in \Delta^{n-1}$, the $R$-orbit of $E_{\lambda}$ is dense in $\mathcal{C} \times \Delta^{n-1}$.

\section{Proof of Theorem 1}

First of all, let us show a relation between the process of renormalization and the key property in Theorem 4 . Let $E:[0,1) \rightarrow[0,1)$ be a minimal interval exchange and let $E_{I}$ be the induced map of $E$ onto $I=[a, b) \subset[0,1)$. Suppose that $L \subset I$ is an interval of continuity of $E_{I}$ and that, for some $x \in L,\left\{E_{I}^{-1}(x), E_{I}(x)\right\} \subset L$. If $r$ is the return time of $L$ to $I$ and $B:=\omega_{0} \omega_{1} \ldots \omega_{r}, \omega_{j} \in\{1,2, \ldots, n\}$, is the itinerary of $x$ until it returns to $L$ (i.e., $E^{j}(x)$ is in the $\omega_{j}$-interval of continuity of $E$ for each $0 \leq j \leq r)$, then the itinerary of every point in $L$, of length $r$, is given by the same word $B$. Therefore as $E_{I}(x) \in L$ and $E_{I}^{-1}(x) \in L$, the itinerary of $x$ associated to $[-r, 2 r]$ will be given by the word

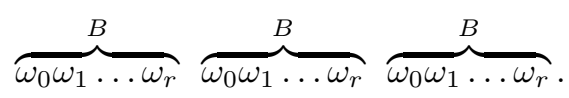

One such point will be called a candidate point in the interval $[a, b)$ for the length $r$. 
Observe that if $E_{I}^{-1}(x), x, E_{I}(x)$, and $E_{I}^{2}(x)$ belong to $L$, then the itinerary of each of the points $y=E^{k}(x), 0 \leq k \leq r$, associated to $[-r, 2 r]$ is of the form $W W W$ where $W$ is the word

$$
\omega_{k} \omega_{k+1} \ldots \omega_{r} \omega_{0} \omega_{1} \ldots \omega_{k-1}
$$

this is, $y$ is also a candidate point for the length $r$. Therefore we have

Lemma 2. Under the assumption right above, assume that

$$
x \in E_{I}^{-2}(L) \cap E_{I}^{-1}(L) \cap L \cap E_{I}(L) .
$$

Then $x, E(x), E^{2}(x), \ldots, E^{r}(x)$ are all candidate points for the length $r$.

Note that if there is a nested sequence of intervals shrinking to a point $x$,

$$
\left[a_{1}, b_{1}\right) \supset\left[a_{2}, b_{2}\right) \supset \cdots \supset\left[a_{k}, b_{k}\right) \supset \ldots,
$$

such that $x$ is a candidate point for each $\left[a_{k}, b_{k}\right)$ for the length $r_{k}$, then necessarily $r_{k} \rightarrow \infty$ as $k \rightarrow \infty$, and the itinerary of $x$ associated to its whole orbit is given by a sequence that satisfies the hypotheses of Theorem 4 .

The periodic iet $P$. Let us consider the following family of periodic iets. Let $n \in \mathbb{N}$ be a natural number and let $\pi \in G_{n}$ be an arbitrary irreducible permutation. Consider the vector

$$
\lambda^{*}:=\left(\frac{1}{n}, \frac{1}{n}, \ldots, \frac{1}{n}\right) \in \mathbb{R}^{n}
$$

then the intervals of continuity of the iet $P:=\left(\pi, \lambda^{*}\right)$ are of the form

$$
I_{k}^{*}:=\left[\frac{k}{n}, \frac{k+1}{n}\right), 0 \leq k \leq n-1,
$$

and $P$ sends any interval $I_{k}^{*}$ onto the interval $I_{\pi(k)}^{*}$. In this way, for each $k \in$ $\{1,2, \ldots, n\}$, there is a positive integer $l_{k}$ such that

$$
P^{l_{k}+1}\left(I_{k}^{*}\right)=I_{k}^{*}
$$

in particular, $P$ is a periodic iet; that is, for some $N \in \mathbb{N}, P^{N}$ is the identity map. We may suppose that $l_{k}$ is the smallest positive integer with this property. Every $x \in I_{k}^{*}$ is a periodic point of period $l_{k}$ and therefore a candidate point for the length $l_{k}$. The induced map of $P$ in $I_{k}^{*}$ is the identity. If $E$ is an iet very close to $P$ and $I$ is an interval of continuity of $E$, the induced map $E_{I}$ in $I$ is very "close to the identity": there is an interval of continuity of $E_{I}$ whose length is very close to that of $I$. By shortening this interval a little we obtain an interval $L$ such that the length of $E_{I}^{-1}(L) \cap E_{I}^{-1}(L) \cap L \cap E_{I}(L)$ is still close to the length of $I$. Lemma 2 guarantees that all the points in this intersection are candidate points for the length $l_{k}$.

By the comment that followed Theorem 4, the proof of Theorem 1 is a consequence of the following proposition.

Proposition 1. Fix an irreducible permutation $\pi \in G_{n}$. Then for almost all $\lambda \in$ $\Delta^{n-1}$ and for almost all $x \in[0,1)$, the coding $\omega_{\lambda}(x)$ satisfies the hypotheses of Theorem 4.

Proof. Consider the periodic iet $P=\left(\pi, \lambda^{*}\right)$, where $\lambda^{*}:=\left(\frac{1}{n}, \frac{1}{n}, \ldots, \frac{1}{n}\right)$, and let $l_{k}, 1 \leq k \leq n-1$, be the smallest positive integer such that $P^{l_{k}}\left(\left[\frac{k}{n}, \frac{k+1}{n}\right)\right)=\left[\frac{k}{n}, \frac{k+1}{n}\right)$.

For any small number $\delta>0$, let $\vartheta(\delta)$ be the set of iets $E=(\pi, \lambda)$ with

$$
\max \left\{\left|\lambda_{i}-\frac{1}{n}\right|: 1 \leq i \leq n\right\}<\delta .
$$


Fix $0<\epsilon<1$. Given a natural number $m$, there exists $\delta_{m}>0$ such that if $E \in \vartheta\left(\delta_{m}\right)$ and $k \in\{1,2, \ldots, n\}$, then the $k^{t h}$-interval of continuity of $E$, denoted by $I_{k}$, contains an interval $L$ such that the sets $E_{I}^{-2}(L), E^{-1}(L)$, and $E(L)$ are subintervals of $I_{k}$ and the interval

$$
M_{k}:=E_{I_{k}}^{-2}(L) \cap E_{I_{k}}^{-1}(L) \cap L \cap E_{I_{k}}(L)
$$

satisfies

$$
\frac{\left|M_{k}\right|}{\left|I_{k}\right|} \geq 1-\frac{\epsilon}{2^{m}}
$$

where $|A|$ denotes the Lebesgue measure of $A$.

Recall that the Rauzy orbit of $E_{\lambda}:=(\pi, \lambda)$ is dense in $\mathcal{C} \times \Delta^{n-1}$ for almost all $\lambda \in \Delta^{n-1}$ (Theorem 6 ). Take an iet $E$ whose Rauzy orbit is dense. Then there are

a1) a nested sequence of half-open intervals

$$
J_{1} \supseteq J_{2} \supseteq \cdots \supseteq J_{m} \supseteq \cdots
$$

given by the Rauzy process of induction,

a2) a sequence of linear bijections $H_{m}: J_{m} \rightarrow[0,1)$, and

a3) an increasing sequence $\left(N_{m}\right)_{m \geq 1}$ of natural numbers such that $E_{m}:=$ $R^{N_{m}}(E)=H_{m} \circ E_{J_{m}} \circ H_{m}^{-1}$ belongs to $\vartheta\left(\delta_{m}\right)$, where $E_{J_{m}}$ is the map induced by $E$ onto $J_{m}$.

Fix $m \in \mathbb{N}$ and, for each $k$ in $\{1,2, \ldots, n\}$, let $I_{k}$ be the $k^{t h}$ interval of continuity of $E_{m}$ and let $M_{k} \subset I_{k}$ be a set that satisfies (4). Let $r_{k}$ be the return time, with respect to $E$, of the interval $I_{k}$ to $J_{m}$. By Lemma 2 , all the iterates

$$
M_{k}, E\left(M_{k}\right), \ldots, E^{r_{k}}\left(M_{k}\right)
$$

are made of candidate points for the length $r_{k}$. As $E$ preserves the Lebesgue measure, each of these sets has Lebesgue measure greater than $1-\epsilon \cdot 2^{-m}$ times the measure of $I_{k}$. This implies that the set of candidate points, for the length $r_{k}$, in the union

$$
\bigcup_{j=1}^{r_{k}} E^{j}\left(I_{k}\right)
$$

is greater than $1-\epsilon \cdot 2^{-m}$ times the Lebesgue measure of this union.

Let $C_{m}$ be the set of candidate points in $[0,1)$ for lengths

$$
r \leq r_{m}:=\max \left\{r_{1}, \ldots, r_{n}\right\} \text {. }
$$

Using the fact that

$$
[0,1)=\bigcup_{k=1}^{n} \bigcup_{j=1}^{r_{k}} E^{j}\left(I_{k}\right)
$$

(see relation (2)), we conclude that $\left|C_{m}\right| \geq 1-\frac{\epsilon}{2^{m}}$.

Now it follows easily that the Lebesgue measure of the intersection

$$
C_{\epsilon}:=\bigcap_{m \geq 1} C_{m}
$$

is greater than $1-\epsilon \sum_{m>1} 2^{-m}=1-\epsilon$. Observe that $\left(r_{m}\right)$ is a strictly increasing sequence and that, for all $m=1,2, \ldots$, every point in $C_{\epsilon}$ is a candidate point for the length $r_{m}$. Therefore all the points in $C_{\epsilon}$ satisfy the condition of Proposition 1. As the number $\epsilon>0$ is arbitrary, Proposition 1 is proved. 


\section{ACKNOWLEDGMENTS}

The third author was partially supported by CNPq. The second author thanks the partial support by FAPESP Grant 03/03107-9 and by CNPqGrant 306992/20035, Brazil. The first author was partially supported by FAPES grant 30898951/2005.

\section{REFERENCES}

[1] Bellissard, J., Iochum, B., Scoppola, E., Testard, D.: Spectral properties of one-dimensional quasi-crystals, Commun. Math. Phys. 125, 527-543 (1989). MR1022526 (90m:82043)

[2] Boshernitzan, M.: A unique ergodicity of minimal symbolic flows with linear block growth, J. Analyse Math. 44, 77-96 (1984/85). MR801288 (87a:28018)

[3] Boshernitzan, M.: A condition for minimal interval exchange maps to be uniquely ergodic, Duke Math. J. 52, 723-752 (1985). MR808101 (87i:28012)

[4] Boshernitzan, M.: A condition for unique ergodicity of minimal symbolic flows, Ergod. Th. \& Dynam. Sys. 12, 425-428 (1992). MR1182655 (93j:58043)

[5] Damanik, D., Lenz, D.: Uniform spectral properties of one dimensional quasicrystals, I. Absence of eigenvalues, Commun. Math. Phys. 207, 687-696 (1999). MR1727235 (2000k:81078)

[6] Damanik, D., Lenz, D.: A condition of Boshernitzan and uniform convergence in the multiplicative ergodic theorem, Duke Math. J. 133, 95-123 (2006). MR2219271 (2007a:37004)

[7] de Oliveira, C. R., Gutierrez, C.: Almost periodic Schrödinger operators along interval exchange transformations, J. Math. Anal. Appl. 283, 570-581 (2003). MR1991829 (2004e:39026)

[8] Delyon, F., Petritis, D.: Absence of localization in a class of Schrödinger operators with quasiperiodic potential, Commun. Math. Phys. 103, 441-444 (1986). MR832920 (87e:81030)

[9] Gordon, A.: On the point spectrum of the one-dimensional Schrödinger operator, Usp. Math. Nauk. 31, 257-258 (1976). MR0458247 (56:16450)

[10] Keane, M.: Interval exchange transformations, Math. Z. 141, 25-31 (1975). MR0357739 (50:10207)

[11] Katok, A., Hasselblatt, B.: Introduction to the Modern Theory of Dynamical Systems. New York: Cambridge Univ. Press, 1995. MR1326374 (96c:58055)

[12] Last, Y., Simon, B.: Eigenfunctions, transfer matrices, and absolutely continuous spectrum of one-dimensional Schrödinger operators, Invent. Math. 135, 329-367 (1999). MR1666767 (2000f:47060)

[13] Masur, H.: Interval exchange transformations and measured foliations, Ann. Math. 115, 169-200 (1982). MR644018 (83e:28012)

[14] Queffélec, M.: Substitution Dynamical Systems - Spectral Analysis, LNM 1294. Berlin: Springer-Verlag, 1987. MR924156 (89g:54094)

[15] Rauzy, G.: Une généralization du développement en fractions continues. Seminaire DelongePisot-Poitou, 18e Année, Vol. 1, (1976-1977), pp. 1501-1515.

[16] Rauzy, G.: Échanges d'intervalles et transformations induites, Acta Arith. 34, 315-328 (1979). MR543205 (82m:10076)

[17] Sütö, A.: The spectrum of a quasiperiodic Schrödinger operator. Commun. Math. Phys. 111, 409-415 (1987). MR900502 (88m:81032)

[18] Veech, W.: Gauss measures for transformations on the space of interval exchange maps, Ann. Math. 115, 201-242 (1982). MR644019 (83g:28036b)

[19] Veech, W.: Boshernitzan criterion for unique ergodicity of an interval exchange transformation, Ergod. Th. \& Dynam. Sys. 7, 149-153 (1987). MR886376 (88d:28022)

Departamento de Matemática, UfeS, Av. F. Ferrari 514, Vitória, ES, 19075-910 BRAZIL

E-mail address: miltonc@cce.ufes.br

Departamento de Matemática, ICMC/USP, CxP 668, São Carlos, SP, 13560-970 BraZil E-mail address: gutp@icmc.usp.br

Departamento de Matemática, UFSCar, São Carlos, SP, 13560-970 Brazil

E-mail address: oliveira@dm.ufscar.br 